\title{
Diagnosis of moyamoya disease using 3-T MRI and MRA: value of cisternal moyamoya vessels.
}

\section{$\operatorname{AUTHOR}(S):$}

Sawada, Takeshi; Yamamoto, Akira; Miki, Yukio; Kikuta, KenIchiro; Okada, Tomohisa; Kanagaki, Mitsunori; Kasahara, Seiko; ... Takahashi, Jun C; Fukuyama, Hidenao; Togashi, Kaori

\section{CITATION:}

Sawada, Takeshi ...[et al]. Diagnosis of moyamoya disease using 3-T MRI and MRA: value of cisternal moyamoya vessels.. Neuroradiology 2012, 54(10): 1089-1097

\section{ISSUE DATE:}

2012-10

URL:

http://hdl.handle.net/2433/162004

\section{RIGHT:}

The final publication is available at www.springerlink.com; This is not the published version. Please cite only the published version.; この論文 は出版社版でありません。引用の際には出版社版をご確認ご利用くだ さい。 


\section{Diagnosis of moyamoya disease using $3 T$ MRI and MRA: Value of cisternal moyamoya vessels}

Takeshi Sawada, $\mathrm{MD}^{11}$, Akira Yamamoto, $\mathrm{MD}, \mathrm{PhD}^{1)}$, Yukio Miki, $\mathrm{MD}, \mathrm{PhD}^{2)}$, Ken-ichiro Kikuta, $\mathrm{MD}, \mathrm{PhD}^{3}$, Tomohisa Okada, $\mathrm{MD}, \mathrm{PhD}^{1}$, Mitsunori Kanagaki, $\mathrm{MD}, \mathrm{PhD}^{1}$, Seiko Kasahara, $\mathrm{MD}, \mathrm{PhD}^{11}$, Susumu Miyamoto, $\mathrm{MD}, \mathrm{PhD}^{4)}$, Jun C. Takahashi, $\mathrm{MD}, \mathrm{PhD}^{4)}$, Hidenao Fukuyama, MD, $\mathrm{PhD}^{5)}$, Kaori Togashi, $\mathrm{MD}, \mathrm{PhD}^{1)}$

1. Department of Diagnostic Imaging and Nuclear Medicine, Kyoto University Graduate School of Medicine, 54 Shogoin-Kawaharacho, Sakyo-ku, Kyoto, 606-8507, Japan

2. Department of Radiology, Osaka City University Graduate School of Medicine 1-4-3, Asahi-machi, Abeno-ku, Osaka 545-8585, Japan

3. Division of Sensory and Locomotor Medicine, Factory of Medical Sciences, University of Fukui, 23-3, Matsuokashimoaizuki, eiheiji-cho, Yoshida-gun, Fukui 910-1193, Japan

4. Department of Neurosurgery, Kyoto University Graduate School of Medicine, 54 Shogoin-Kawaharacho, Sakyo-ku, Kyoto, 606-8507, Japan

5. Human Brain Research Center, Kyoto University Graduate School of Medicine, 54 Shogoin-Kawaharacho, Sakyo-ku, Kyoto, 606-8507, Japan

Corresponding Author: Akira Yamamoto, $\mathrm{MD}, \mathrm{PhD}$

Tel: +81-75-751-3760

Fax: +81-75-771-9709

e-mail: yakira@kuhp.kyoto-u.ac.jp 


\begin{abstract}
Introduction The purpose of this study was to propose a new MR criteria of diagnosing MMD from cisternal MMVs on 3T MRI/MRA and compare the diagnostic accuracy of the existing MR criteria and the proposed MR criteria.

Methods Participants comprised 20 consecutive patients with MMD (4 males, 16 females) diagnosed clinically using conventional angiography, and 20 controls (13 male and 7 female arteriosclerosis patients). In these participants, 3T MRI/MRA was evaluated by the existing MR criteria, which use MMVs in basal ganglia and the proposed MR criteria, which use cisternal MMVs, then these two criteria were statistically compared by McNemar's test.

Results Diagnostic accuracy was $62.5 \%$ with the existing MR criteria and 97.5\% with the proposed MR criteria. The proposed MR criteria was more sensitive (1.00) than the existing MR criteria (0.45), but less specific (0.95) than the existing MR criteria (1.00).

Conclusion The proposed MR criteria using cisternal MMVs showed significantly higher diagnostic accuracy than the existing MR criteria. We believe that our proposed MR criteria will be beneficial for diagnosing MMD.
\end{abstract}




\section{KEYWORDS}

cisternal moyamoya vessels, moyamoya disease, magnetic resonance angiography, magnetic resonance imaging, T2-weighted imaging 
Abbreviations

ACA

AUC

CSF

FSE

ICA

MCA

MIP

MMD

MMVs

MR

MRA

MRI

ROC

SNR

SWI

T2WI

TOF anterior cerebral artery

area under the curve

cerebrospinal fluid

fast spin echo

internal carotid artery

middle cerebral artery

maximum intensity projection

moyamoya disease

moyamoya vessels

magnetic resonance

magnetic resonance angiography

magnetic resonance imaging

receiver operating characteristic

signal-to-noise ratio

susceptibility-weighted imaging

T2-weighted imaging

time-of-flight 


\section{INTRODUCTION}

MMD is a relatively rare disorder in which distal portions of the ICAs and/or proximal portions of the ACA and/or MCA become progressively steno-occlusive with secondary formation of collateral vessels and abnormal vascular network called MMVs[1]. This disease occurs predominantly in Japanese, but occurrence has also been reported in other countries[2-3]. The etiology of MMD has been unknown for long, but recently its responsible genes are being identified[4]. There are reported links between moyamoya-like state and a wide variety of other disorders such as radiation arteritis, Down's syndrome and so on, they are excluded clinically[5]. By the finding of conventional angiography, disease severity is frequently classified into one of six progressive stage (Suzuki's stage)[1]. MMD is well known to cause transient ischemic attacks, cerebral infarction, or intracranial bleeding in both children and adults[6-7]. Intracranial bleeding, in particular, often results in poor outcomes[1]. Cases with relatively mild and slow progression are usually treated more conservatively, while cases with faster progression are treated surgically, such as with vascular bypass methods[2]. Treatment of MMD depends on the possibility of faster deterioration, so frequent follow-up imaging as well as accurate diagnosis and staging of the disease on each examination is indispensable in the clinical situation.

MMD has been diagnosed using diagnostic criteria that apply results from conventional angiography and MRI/MRA independently[5]. In the existing guideline, conventional angiography still remains to be the gold standard for the diagnosis of MMD in principle[8-9], and the relatively large number of patients that do not fulfill the diagnostic criteria from MRI/MRA need diagnostic conventional angiography[5,10-11]. Conventional angiography can visualize stenosis of both normal and MMVs more clearly and definitively, therefore enabling more precise evaluation of vessels, which is necessary for surgical treatment. One drawback of the existing diagnostic criteria using conventional angiography is the possibility of unwanted side effects such as contrast media allergy which might be serious in some cases[12]. Another drawback of the existing diagnostic criteria is the technical difficulty of conventional angiography in pediatric cases due to the narrow artery and need for sedation.

Nowadays, 3T MR is widely used in clinical situations and has been 
reported to show superiority to $1.0 / 1.5 \mathrm{~T} \mathrm{MR}$ in the diagnosis of various neurological disorders [13-15]. The higher spatial resolution and SNR of 3T MR are useful for evaluating the fine features of MMD. MMVs have been reported to be better delineated with MRA at 3T than at 1.5T[15]. For microbleeds in MMD, representing an important factor for prognosis[16], SWI has been reported to offer better detection at $3 \mathrm{~T}$ than $1.5 \mathrm{~T}$ MRI[17]. In the clinical situation, MRI/MRA findings have been used for diagnosis of MMD, such as cisternal MMV and the FLAIR ivy sign [18-19]. Among these fine features of MMD, the cisternal MMVs visualized as a distinct feature of MMD on $3 \mathrm{~T}$ MR might be beneficial for diagnosing MMD. These fine features of MMD visualized by recent MR technique have been available by now, therefore an updated guideline which reflects these benefits has been needed. This study proposes a new diagnostic criteria for MMD using cisternal MMVs evaluated by routine clinical 3T MRI/MRA. We hypothesized that the proposed MR diagnostic criteria using 3T MR can diagnose MMD more accurately by evaluating cisternal MMVs than the existing MR criteria, which evaluates MMVs in the basal ganglia. The purpose of this study was to evaluate the diagnostic accuracy of the proposed MR criteria and the existing MR criteria using 3T MRI/MRA. 


\section{MATERIALS AND METHODS}

\section{Patients}

All study protocols were approved by the local ethics committee and written informed consent was obtained from all patients or their parents. The study included 20 consecutive patients with MMD from March 2004 to April 2008 (4 male, 16 female; mean age 26.3 years; range, 2-58 years) and 20 controls (13 male and 7 female arteriosclerosis patients; mean age, 64.7 years; range, 27-87 years). All MMD patients had been diagnosed clinically using the existing diagnostic criteria, including conventional angiography. Suzuki's stages of MMD patients are shown in Table 1. Cases of unilateral MMD were not included in this study. Control arteriosclerosis patients showed multi-vessel stenosis. Four cases showed $\geq 3$ vessel stenoses, 8 cases showed 2 vessel stenoses, and 8 cases showed stenosis of 1 vessel.

\section{Image acquisition}

All patients and controls underwent 3D TOF MRA and FSE T2WI using a 3T MR scanner (Magnetom Trio; Siemens, Erlangen, Germany). A dedicated head array coil was used for image acquisition. The following parameters were used for TOF MRA: repetition time, $22 \mathrm{~ms}$; echo time, $3.7 \mathrm{~ms}$; flip angle, $20^{\circ}$; slice thickness, $0.7 \mathrm{~mm}$; slab thickness, 33.6mm; number of slabs, 3; field of view, $200 \times 150$ $\mathrm{mm}$; matrix, $320 \times 240$; voxel size, $0.625 \times 0.625 \times 0.7 \mathrm{~mm}$; and acquisition time, 5 min $51 \mathrm{~s}$. The following parameters were used for FSE T2WI: repetition time, $3200 \mathrm{~ms}$; echo time, $79 \mathrm{~ms}$; echo trains per slice, 14; slice thickness, $3 \mathrm{~mm}$; slice gap, $1 \mathrm{~mm}$; field of view, $220 \times 176 \mathrm{~mm}$; matrix, $448 \times 360$; voxel size, $0.6 \times 0.5$ $\times 3 \mathrm{~mm}$; and acquisition time, $1 \mathrm{~min} 44 \mathrm{sec}$.

\section{Diagnostic methods}

The existing MR criteria for diagnosis of MMD using MRI/MRA[5] and the proposed MR criteria for diagnosis of MMD using 3T MRI/MRA are shown in Table 2, Fig. 1 and 2.

In Sylvian valley, T2WI shows cisternal MMVs as worm-like structures 
and a network of numerous randomly formed flow voids in axial slices (Fig. 3). Source images from TOF MRA also show MMVs as a network of numerous randomly formed high signal intensity structures in the cisternal low signal intensity (Fig. 4). In this study, cisternal MMVs were evaluated on T2WI and TOF MRA with source images.

\section{Image evaluation}

Two board-certified neuroradiologists (T.S. and A.Y. over 8 years experience) evaluated T2WI and TOF MRA using the existing and proposed MR criteria. Evaluation based on MRI/MRA for each side was classified into 5 categories (5, absolutely positive; 4 , probably positive; 3 , unclear; 2 , probably negative; and 1 , absolutely negative).

The procedure for evaluation using the existing MR criteria is as follows: a) evaluation of whether stenosis exists at the ICA and/or ACA, and/or MCA on the right side; b) evaluation of MMVs in the basal ganglia on T2WI(Fig. 2) on the right side; and c) repetition of the same evaluations for the left side.

The procedure for evaluation using the proposed MR criteria is as follows: a) evaluation of whether stenosis exists at the ICA and/or ACA, and/or MCA on the right side; b) evaluation of MMVs in Sylvian valley(Fig. 3,4) on the right side; and c) repetition of the same evaluation on the left side.

In this study, evaluation was performed on the right and left sides separately, because the degree of stenosis of arteries and formation of MMVs are not symmetrical in all MMD patients[20]. In the existing MR criteria and the proposed MR criteria, the concordance rate of the scores by the two neuroradiologists was evaluated by the Kappa coefficient. If the scores for each side did not fully agree, the scores were decided by consensus. The diagnostic category for the patient was defined as the average of category scores of the left and right sides. Sample images of a MMD patient are shown in Fig. 5. The diagnostic category by the existing MR criteria was 1 , and that by the proposed MR criteria was 5 . 


\section{Statistical analysis}

As the first analysis, ROC curves for both the existing and proposed MR criteria were used for statistical evaluation of diagnostic accuracy. AUC for each criteria was then calculated and comparisons of diagnostic accuracy for the existing and proposed MR criteria were performed using Hanlay's method, with values of $\mathrm{p}<0.05$ considered significant[21].

As the second analysis, cut-off points were calculated for each criteria to maximize sensitivity and specificity. Using these cut-off points for each criteria, diagnostic accuracies of the two criteria for clinical diagnosis were calculated according to results from conventional angiography and compared using McNemar's test[22].

As the third analysis, to avoid calculation bias from comparing two criteria with separate cut-off points, a common cut-off point was determined at the median of the range (category 3). Categories $<3$ were considered as not MMD and categories $\geq 3$ as MMD, with category 3 included in MMD to maximize the sensitivity of the diagnostic method. With this common cut-off point, diagnostic accuracies of two criteria for clinical diagnosis using conventional angiography were calculated and compared using McNemar's test[22]. 


\section{RESULTS}

The Kappa coefficient of the scores of the two neuroradiologists by the existing MR criteria was 0.63 , and that by the proposed criteria was 0.89 . ROC curves for the two criteria are shown in Fig. 6. The diagnostic categories for MMD patients and controls are shown in Table 1. Standard deviations for the existing MR criteria were 0.71 in MMD patients and 0 in controls, and those for the proposed MR criteria were 0.26 in MMD patients and 1.16 in controls. Median values for the existing MR criteria were 1 in MMD cases and 1 in controls, and those for the proposed MR criteria were 5 in MMD patients and 1 in controls.

AUC of the existing MR criteria was 0.725 (two-sided 95\% confidence interval: $0.567-0.883$ ). AUC of the proposed MR criteria was 0.999 (two-sided 95\% confidence interval: 0.988-1.000). A comparative test using Hanlay's method showed the difference of two AUCs was 0.274 , with a standard error of 0.080 , and a correlation coefficient between the two AUCs of 3.403. A significant difference between the two criteria was identified $(\mathrm{p}=0.0007)$.

In the second analysis, cut-off points were calculated for each criteria to maximize sensitivity and specificity using ROC curves. The cut-off point for the existing MR criteria was 1.5 (sensitivity, 0.45 ; specificity, 1.00), compared to 3.25 (sensitivity, 1.00; specificity, 0.95) for the proposed MR criteria. Using conventional angiography as the gold standard, accuracies of the two criteria with each cut-off point were $72.5 \%$ for the existing MR criteria and $97.5 \%$ for the proposed MR criteria. McNemar's test showed a significant difference between the two criteria $(p=0.006)$.

In the third analysis, the cut-off point for the existing and proposed MR criteria was the common cut-off point 3 . Using conventional angiography as the gold standard, accuracies of the two criteria with the common cut-off point were $62.5 \%$ for the existing MR criteria and $97.5 \%$ for the proposed MR criteria. McNemar's test showed a significant difference between the two criteria $(\mathrm{p}=0.001)$.

The results of the second and third analysis are also shown in Table 3. 


\section{DISCUSSION}

This study evaluated 3T MRI/MRA in 20 MMD patients and 20 controls using the existing and proposed MR criteria for diagnosis of MMD. AUC of ROC curves for these two criteria showed a significant difference. Accuracies of the existing and proposed MR criteria showed a significant difference according to McNemar's test using each cut-off point and common cut-off point. The proposed MR criteria using 3T MRI/MRA showed superior diagnostic accuracy for MMD.

The existing diagnostic criteria of MMD were defined by the Research Committee on Spontaneous Occlusions of the Circle of Willis (MMD) of the Ministry of Health and Welfare of Japan in 1997[5]. At that time, 1.0T or 1.5T MR were widely used and 3T MR were not widely available in clinical situations. In the evaluation of MMD patients, features of vessels delineated by MRI/MRA using 1.0/1.5T MR might be suboptimal for definitive diagnosis in more than a few cases[11]. Substantial cases were unable to be diagnosed using MRI/MRA alone, so conventional angiography has been required as the gold standard for definite diagnosis of MMD in not a few patients, including pediatric cases[23].

If the definite standard with high accuracy of diagnosis using the relatively less-invasive MR had settled, more patients could skip conventional angiography. This would prove beneficial for MMD patients, particularly for children. Several studies have discussed the diagnosis of MMD using MRI/MRA, mainly using $1.5 \mathrm{~T}$ MR[11,24-27]. Although 1.5T MR can be used for diagnosing MMD in the clinical setting, the accuracy of diagnosis has been suboptimal due to the intrinsic difficulties of acquiring higher spatial resolution and higher SNR[28]. In many cases, conventional angiography has been used as the gold standard for clinical diagnosis of MMD under the existing diagnostic criteria[8-10].

Cisternal MMVs, one of the fine features of MMD, are defined as tortuous small vessels in Sylvian valley other than the MCA and its perforators and are visualized as worm-like structures on axial slices in MRI/MRA[18,29] (Fig. 3,4). These cisternal MMVs have been visualized using high-resolution T2WI with constructive interference in steady state imaging on $1.5 \mathrm{~T}$ systems and may be beneficial in diagnosing MMD using MRI[30]. However, this sophisticated technique requires a relatively long scan time, and has thus been difficult to use in screening and frequent follow-up examinations.

Nowadays, 3T MR has been introduced widely into clinical situations. 
Various features of MMD have been reported to be more clearly apparent with 3T MR than with 1.5T MR, including vascular abnormalities on MRA and microbleeds on SWI[15,17]. Some reports have noted that 3T MRI/MRA visualizes more fine features of MMVs than MRI/MRA at 1.0/1.5T[14-15]. As 3T MRI offers a higher SNR than 1.0/1.5T MR, a higher spatial resolution on T2WI can be achieved. Also, 3T MR have a longer longitudinal relaxation time than 1.0/1.5T MR, allowing improved T1 contrast and a higher signal on TOF MRA. These superior properties of 3T MRI/MRA might have performed a significant role in improving the accuracy of diagnosing MMD in this study.

With the existing MR criteria, MMV is evaluated as flow voids in the basal ganglia on T2WI. On routine clinical 3T MR images, flow voids in the basal ganglia may be difficult to detect. Compared to $1.5 \mathrm{~T}$ MRI, the basal ganglia sometimes show as areas of low signal intensity on 3T MRI due to the rich iron content[31], so low signal flow voids might be difficult to detect in the basal ganglia. Conversely, with the proposed MR criteria, MMVs were steadily evaluated in Sylvian valley, where low-signal vascular flow voids are more readily apparent against the background of high signal intensity CSF on T2WI [29]. In addition, 3T MRA showed MMVs more clearly in both the basal ganglia and Sylvian valley, due to the longer longitudinal relaxation time[15]. In the present study, T2WI and MRA were evaluated together in both the existing and proposed MR criteria, which may prevent influences of artifacts such as CSF flow void in Sylvian valley complementarily and facilitate evaluation of fine features of MMVs in the proposed MR criteria.

If MMD can be definitively diagnosed using only 3T MRI/MRA, conventional angiography can be skipped. This may be beneficial for the first diagnosis of MMD, as the procedure for conventional angiography can have unwanted side effects[12]. In addition, this may be beneficial for patients with slow progression, who can be followed up using MRI/MRA alone and treated conservatively without surgical therapy unless frequent imaging examinations show deterioration [32]. Diagnosis of MMD using 3T MRI/MRA alone may thus be beneficial for accurate first diagnosis and subsequent imaging follow-ups for early treatment[33-34].

Several limitations must be considered when interpreting the results of this study. First, age-matched controls were not enrolled in the study. This is 
because MMD patients in this study were younger than the control patients with arteriosclerosis. The incidence of MMD shows peaks in two age groups: children at approximately 5 years old and adults in their mid-40s[3,35]. Second, we used cisternal MMVs in Sylvian valley for diagnosis, but MMVs do not become obvious at stage 1, and vanish at stage 5-6[1]. The proposed MR criteria may not work in such low stage and higher-stage cases. Suzuki's stages 1 and 2 are considered as the early stages of MMD. Stages 3 and 4, which are the most frequently observed stages, are clinically important and have to be clinically discriminated. Suzuki's stages 5 and 6 are considered as the final stages of $\operatorname{MMD}[1,35]$. And onset of the disease at stage 5 or 6 is rare, so evaluation of cisternal MMVs may be feasible in many cases. Diagnosis of stage 1 cases by MRI/MRA is one of our future tasks and under way. 


\section{CONCLUSION}

This study evaluated the accuracy of the proposed MR criteria for diagnosing MMD using cisternal MMVs visualized by 3T MRI/MRA. The diagnostic accuracy of the proposed MR criteria was higher than that of the existing MR criteria. We believe that the proposed MR criteria using 3T MRI/MRA will prove beneficial for diagnosing MMD.

\section{CONFLICT OF INTEREST}

We declare that we have no conflict of interest. 


\section{REFERENCES}

1. Suzuki J, Takaku A (1969) Cerebrovascular "moyamoya" disease. Disease showing abnormal net-like vessels in base of brain. Arch Neurol 20 (3):288-299

2. Burke GM, Burke AM, Sherma AK, Hurley MC, Batjer HH, Bendok BR (2009) Moyamoya disease: a summary. Neurosurg Focus 26 (4):E11. doi:10.3171/2009.1.FOCUS08310

3. Takahashi JC, Miyamoto S (2010) Moyamoya disease: recent progress and outlook. Neurol Med Chir (Tokyo) 50 (9):824-832. doi:JST.JSTAGE/nmc/50.824 [pii]

4. Liu W, Morito D, Takashima S, Mineharu Y, Kobayashi H, Hitomi T, Hashikata H, Matsuura N, Yamazaki S, Toyoda A, Kikuta K, Takagi Y, Harada KH, Fujiyama A, Herzig R, Krischek B, Zou L, Kim JE, Kitakaze M, Miyamoto S, Nagata K, Hashimoto N, Koizumi A (2011) Identification of RNF213 as a Susceptibility Gene for Moyamoya Disease and Its Possible Role in Vascular Development. PLoS One 6 (7):e22542. doi:10.1371/journal.pone.0022542

PONE-D-10-04031 [pii]

5. Fukui M (1997) Guidelines for the diagnosis and treatment of spontaneous occlusion of the circle of Willis ('moyamoya' disease). Research Committee on Spontaneous Occlusion of the Circle of Willis (Moyamoya Disease) of the Ministry of Health and Welfare, Japan. Clin Neurol Neurosurg 99 Suppl 2:S238-240

6. Jones BP, Ganesan V, Saunders DE, Chong WK (2010) Imaging in childhood arterial ischaemic stroke. Neuroradiology 52 (6):577-589. doi:10.1007/s00234-0100704-7

7. Serdaru M, Gray F, Merland JJ, Escourolle R, Grumbach R (1979) Moyamoya disease and intracerebral hematoma. Clinical pathological report. Neuroradiology 18 (1):47-52

8. Currie S, Raghavan A, Batty R, Connolly DJ, Griffiths PD (2011) Childhood moyamoya disease and moyamoya syndrome: a pictorial review. Pediatr Neurol 44 (6):401-413. doi:S0887-8994(11)00103-2 [pii]

10.1016/j.pediatrneurol.2011.02.007

9. Takanashi J (2011) Moyamoya disease in children. Brain Dev 33 (3):229-234. doi:S0387-7604(10)00231-7 [pii]

10.1016/j.braindev.2010.09.003

10. Lee DJ, Liebeskind DS (2011) Characterization of inpatient moyamoya in the United States: 1988-2004. Front Neurol 2:43. doi:10.3389/fneur.2011.00043

11. Houkin K, Aoki T, Takahashi A, Abe H (1994) Diagnosis of moyamoya disease with magnetic resonance angiography. Stroke 25 (11):2159-2164

12. Maki Y, Enomoto T (1988) Moyamoya disease. Childs Nerv Syst 4 (4):204-212

13. Tanenbaum LN (2004) 3-T MR imaging: ready for clinical practice. AJNR Am J Neuroradiol 25 (9):1626-1627; author reply 1629. doi:25/9/1626 [pii]

14. Thomas SD, Al-Kwifi O, Emery DJ, Wilman AH (2002) Application of magnetization transfer at $3.0 \mathrm{~T}$ in three-dimensional time-of-flight magnetic resonance angiography of the intracranial arteries. Journal of Magnetic Resonance Imaging 15 (4):479-483. doi:Doi 10.1002/Jmri.10085

15. Fushimi Y, Miki Y, Kikuta K, Okada T, Kanagaki M, Yamamoto A, Nozaki K, Hashimoto N, Hanakawa T, Fukuyama H, Togashi K (2006) Comparison of 3.0- and 1.5-T three-dimensional time-of-flight $\mathrm{MR}$ angiography in moyamoya disease: preliminary experience. Radiology 239 (1):232-237. doi:2383042020 [pii]

10.1148/radiol.2383042020

16. Kikuta K, Takagi Y, Nozaki K, Hanakawa T, Okada T, Mikuni N, Miki Y, Fushmi Y, Yamamoto A, Yamada K, Fukuyama H, Hashimoto N (2005) Asymptomatic microbleeds in moyamoya disease: T2*-weighted gradient-echo magnetic resonance imaging study. J Neurosurg 102 (3):470-475. doi:10.3171/jns.2005.102.3.0470

17. Mori N, Miki Y, Kikuta K, Fushimi Y, Okada T, Urayama S, Sawamoto N, Fukuyama H, Hashimoto N, Togashi K (2008) Microbleeds in moyamoya disease: susceptibility-weighted imaging versus $\mathrm{T} 2{ }^{*}$-weighted imaging at 3 Tesla. Invest Radiol 43 (8):574-579. doi:10.1097/RLI.0b013e31817fb432

00004424-200808000-00005 [pii]

18. Illner A (2009) Moyamoya. In: Osborn AG, Salzman KL, Barkovich AJ (eds) 
Diagnostic imaging: Brain. 2nd edn. Lippincott Williams \& Wilkins, Salt Lake City, Utah, pp I-4-46-49

19. Maeda M, Tsuchida C (1999) "Ivy sign" on fluid-attenuated inversion-recovery images in childhood moyamoya disease. Am J Neuroradiol 20 (10):1836-1838

20. Guzman R, Lee M, Achrol A, Bell-Stephens T, Kelly M, Do HM, Marks MP, Steinberg GK (2009) Clinical outcome after 450 revascularization procedures for moyamoya disease. Clinical article. J Neurosurg 111 (5):927-935. doi:10.3171/2009.4.JNS081649

21. Hanley JA, Mcneil BJ (1983) A Method of Comparing the Areas under Receiver Operating Characteristic Curves Derived from the Same Cases. Radiology 148 (3):839-843

22. McNemar Q (1947) Note on the Sampling Error of the Difference between Correlated Proportions or Percentages. Psychometrika 12 (2):153-157

23. Fukui M (1997) Current state of study on moyamoya disease in Japan. Surg Neurol 47 (2):138-143. doi:S0090-3019(96)00358-8 [pii]

24. Fujisawa I, Asato R, Nishimura K, Togashi K, Itoh K, Noma S, Sagoh T, Minami S, Nakano Y, Yonekawa Y, et al. (1987) Moyamoya disease: MR imaging. Radiology 164 (1):103-105

25. Yamada I, Matsushima Y, Suzuki S (1992) Moyamoya disease: diagnosis with three-dimensional time-of-flight MR angiography. Radiology 184 (3):773-778

26. Battistella PA, Carollo C, Pellegrino PA, Soriani S, Scarpa P (1995) Magnetic resonance angiography in moyamoya disease. Childs Nerv Syst 11 (6):329-334

27. Houkin K, Nakayama N, Kuroda S, Nonaka T, Shonai T, Yoshimoto T (2005) Novel magnetic resonance angiography stage grading for moyamoya disease. Cerebrovasc Dis 20 (5):347-354. doi:CED2005020005347 [pii]

$10.1159 / 000087935$

28. Yamada I, Suzuki S, Matsushima Y (1995) Moyamoya disease: diagnostic accuracy of MRI. Neuroradiology 37 (5):356-361

29. Bacigaluppi S, Dehdashti AR, Agid R, Krings T, Tymianski M, Mikulis DJ (2009) The contribution of imaging in diagnosis, preoperative assessment, and follow-up of moyamoya disease: a review. Neurosurg Focus 26 (4):E3. doi:10.3171/2009.01.FOCUS08296

30. Komiyama M, Ishiguro T, Nishikawa M, Yasui T, Morikawa T, Kitano S, Sakamoto H (2002) Constructive interference in steady state imaging of moyamoya disease. Neurol Med Chir (Tokyo) 42 (1):11-16; discussion 17. doi:JST.JSTAGE/nmc/42.11 [pii]

31. Yao B, Li TQ, van Gelderen P, Shmueli K, de Zwart JA, Duyn JH (2009) Susceptibility contrast in high field MRI of human brain as a function of tissue iron content. Neuroimage 44 (4):1259-1266. doi:10.1016/j.neuroimage.2008.10.029

32. Hallemeier CL, Rich KM, Grubb RL, Chicoine MR, Moran CJ, Cross DT, Zipfel GJ, Dacey RG, Derdeyn CP (2006) Clinical features and outcome in North American adults with moyamoya phenomenon. Stroke 37 (6):1490-1496. doi:Doi 10.1161/01.Str.0000221787.70503.Ca

33. Kim SK, Seol HJ, Cho BK, Hwang YS, Lee DS, Wang KC (2004) Moyamoya disease among young patients: Its aggressive clinical course and the role of active $\begin{array}{llll}\text { surgical treatment. Neurosurgery } 54 & (4): 840-845 . & \text { doi:Doi }\end{array}$ 10.1227/01.Neu.0000114140.41509.14

34. Scott RM, Smith JL, Robertson RL, Madsen JR, Soriano SG, Rockoff MA (2004) Long-term outcome in children with moyamoya syndrome after cranial revascularization by pial synangiosis. Journal of Neurosurgery 100 (2):142-149 35. Scott RM, Smith ER (2009) Moyamoya disease and moyamoya syndrome. N Engl J Med 360 (12):1226-1237. doi:360/12/1226 [pii]

10.1056/NEJMra0804622 


\section{FIGURE LEGENDS}

Fig. 1: MRA MIP of MMD showing stenosis of bilateral ICAs.

Fig. 2: Axial FSE T2WI shows MMVs at the level of the basal ganglia. Flow voids indicating abnormal vascular networks are visualized at bilateral putamina.

Fig. 3: Axial FSE T2WI shows MMVs at the level of Sylvian valley. Flow voids of MMVs were apparent in bilateral Sylvian valleys.

Fig. 4: MRA source image also shows MMVs at the level of Sylvian valley. MMVs were apparent in bilateral Sylvian valleys as high signal intensity.

Fig. 5: MRA MIP, T2WI and MRA source images from a patient with MMD (category 5 by the proposed MR criteria; category 1 by the existing MR criteria). a) MRA MIP. b) T2WI at the level of Sylvian valley . c) T2WI at the level of the basal ganglia. d) MRA source image at the level of Sylvian valley. e) MRA source image at the level of the basal ganglia.

Fig. 6: ROC curves of the two criteria. 
Table 1: The diagnostic category of MMD patients and controls

Existing MR criteria

\begin{tabular}{|c|c|c|c|c|c|}
\hline Age, Sex & Diagnosis & Category & Category & $\mathrm{Rt}$ & $\mathrm{Lt}$ \\
\hline $02 Y, F$ & MMD & 4 & 5 & 4 & 4 \\
\hline 06Y, F & MMD & 3 & 5 & 3 & 4 \\
\hline 06Y, F & MMD & 2.5 & 4.5 & 3 & 2 \\
\hline 07Y, F & MMD & 2 & 5 & 2 & 3 \\
\hline 08Y, F & MMD & 3 & 5 & 2 & 4 \\
\hline 08Y, M & MMD & 5 & 5 & 4 & 4 \\
\hline $13 \mathrm{Y}, \mathrm{F}$ & MMD & 1 & 5 & 2 & 3 \\
\hline $14 \mathrm{Y}, \mathrm{F}$ & MMD & 1 & 5 & 2 & 2 \\
\hline $21 \mathrm{Y}, \mathrm{F}$ & MMD & 1 & 4.5 & 2 & 2 \\
\hline $21 Y, F$ & MMD & 2.5 & 5 & 4 & 4 \\
\hline $22 \mathrm{Y}, \mathrm{F}$ & MMD & 1 & 5 & 4 & 4 \\
\hline $31 \mathrm{Y}, \mathrm{F}$ & MMD & 2 & 5 & 3 & 3 \\
\hline $32 \mathrm{Y}, \mathrm{F}$ & MMD & 1 & 5 & 3 & 2 \\
\hline $35 \mathrm{Y}, \mathrm{F}$ & MMD & 1 & 4 & 2 & 2 \\
\hline $47 \mathrm{Y}, \mathrm{F}$ & MMD & 3 & 5 & 3 & 3 \\
\hline $48 \mathrm{Y}, \mathrm{F}$ & MMD & 1 & 5 & 4 & 3 \\
\hline 49Y, F & MMD & 1 & 5 & 3 & 3 \\
\hline 49Y, M & MMD & 1 & 4.5 & 2 & 2 \\
\hline $50 \mathrm{Y}, \mathrm{M}$ & MMD & 1 & 5 & 3 & 3 \\
\hline $58 \mathrm{Y}, \mathrm{M}$ & MMD & 1 & 5 & 3 & 3 \\
\hline $27 \mathrm{Y}, \mathrm{M}$ & CTR & 1 & 4 & & \\
\hline $30 \mathrm{Y}, \mathrm{M}$ & CTR & 1 & 1 & & \\
\hline $41 \mathrm{Y}, \mathrm{F}$ & CTR & 1 & 1 & & \\
\hline $42 \mathrm{Y}, \mathrm{F}$ & CTR & 1 & 1 & & \\
\hline $61 \mathrm{Y}, \mathrm{F}$ & CTR & 1 & 1 & & \\
\hline $65 \mathrm{Y}, \mathrm{M}$ & CTR & 1 & 1 & & \\
\hline $66 \mathrm{Y}, \mathrm{M}$ & CTR & 1 & 1 & & \\
\hline $66 \mathrm{Y}, \mathrm{M}$ & CTR & 1 & 1 & & \\
\hline $66 \mathrm{Y}, \mathrm{M}$ & CTR & 1 & 1 & & \\
\hline 69Y, F & CTR & 1 & 1 & & \\
\hline $70 Y, F$ & CTR & 1 & 1 & & \\
\hline $73 \mathrm{Y}, \mathrm{M}$ & CTR & 1 & 1 & & \\
\hline $74 \mathrm{Y}, \mathrm{M}$ & CTR & 1 & 1 & & \\
\hline $74 Y, F$ & CTR & 1 & 1 & & \\
\hline $76 \mathrm{Y}, \mathrm{M}$ & CTR & 1 & 2.5 & & \\
\hline 76Y, M & CTR & 1 & 1 & & \\
\hline 76Y, F & CTR & 1 & 1 & & \\
\hline $77 \mathrm{Y}, \mathrm{M}$ & CTR & 1 & 1 & & \\
\hline $78 \mathrm{Y}, \mathrm{M}$ & CTR & 1 & 1 & & \\
\hline 87Y, M & CTR & 1 & 1 & & \\
\hline
\end{tabular}

$\mathrm{MMD}=$ moyamoya disease, $\mathrm{CTR}=$ control 
Table 2: The existing and proposed MR criteria for diagnosing MMD

\section{The existing MR criteria[5]}

When MRI and MRA clearly demonstrate all the below findings, conventional cerebral angiography is not mandatory.

1. Stenosis or occlusion at the terminal portion of the ICA and/or at the proximal portion of the ACA and/or the MCA(Fig. 1).

2. An abnormal vascular network in the basal ganglia on MRA. An abnormal vascular network can be diagnosed when more than two flow voids are seen on one side of the basal ganglia on MRI(Fig. 2).

3. Bilateral appearance of (1) and (2).

\section{The proposed MR criteria}

1. Stenosis or occlusion at the terminal portion of the ICA and/or at the proximal portion of the ACA and/or the MCA. (Fig. 1).

2. An abnormal vascular network of cisternal MMVs apparent in bilateral Sylvian valleys on T2WI or MRA(Fig. 3,4).

3. Bilateral appearance of (1) and (2). 
Table 3: The results of the second and third analysis

\begin{tabular}{|l|l|l|l|l|l|}
\hline \multicolumn{2}{|c|}{} & cut-off point & Sensitivity & Specificity & Accuracy \\
\hline \multirow{2}{*}{ Second analysis } & Existing MR criteria & 1.5 & 0.45 & 1.00 & $72.5 \%$ \\
\cline { 2 - 6 } & Proposed MR criteria & 3.25 & 1.00 & 0.95 & $97.5 \%$ \\
\hline \multirow{2}{*}{ Third analysis } & Existing MR criteria & 3 & & & $62.5 \%$ \\
\cline { 2 - 6 } & Proposed MR criteria & & & & $97.5 \%$ \\
\hline
\end{tabular}




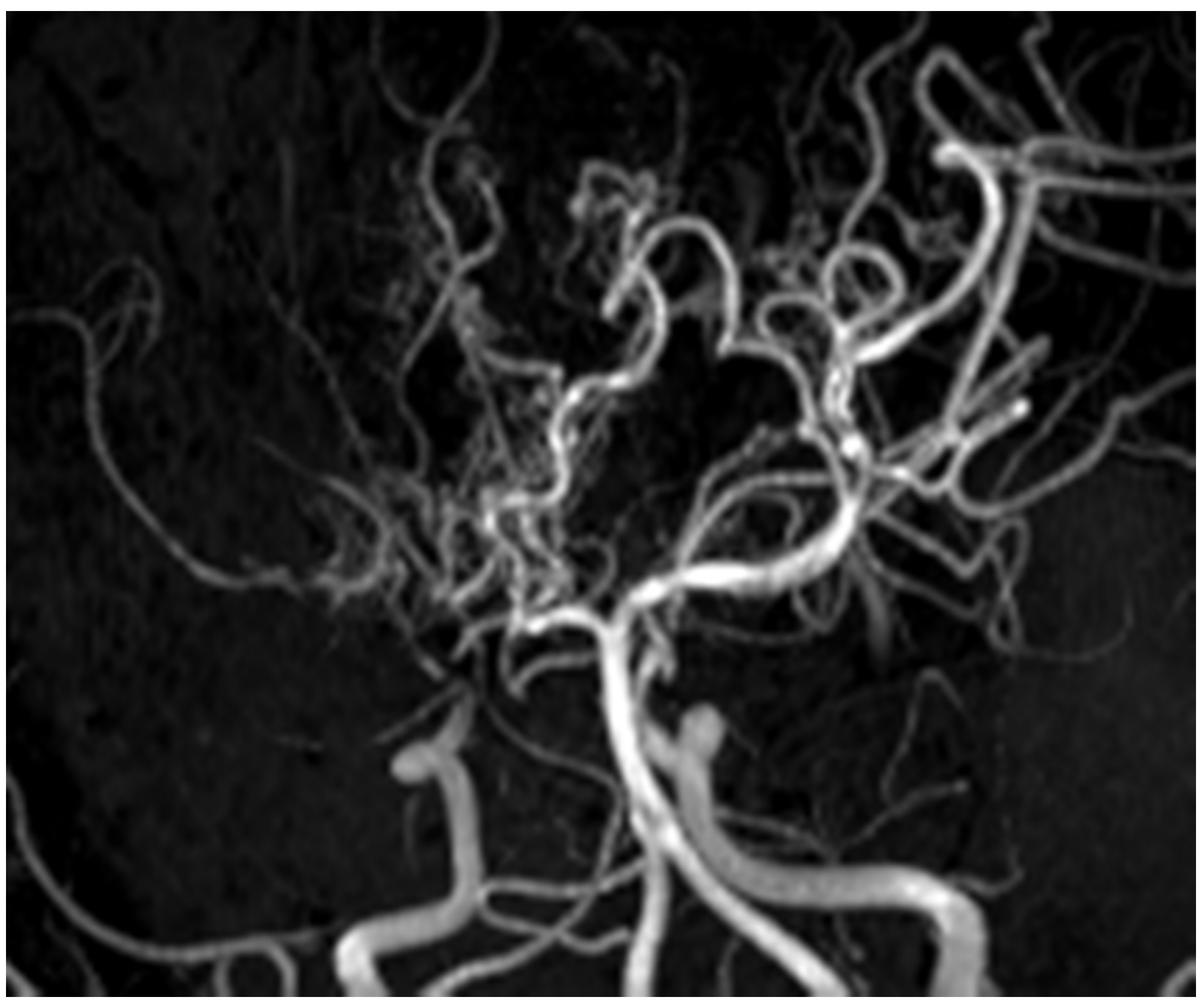

Fig. 1 


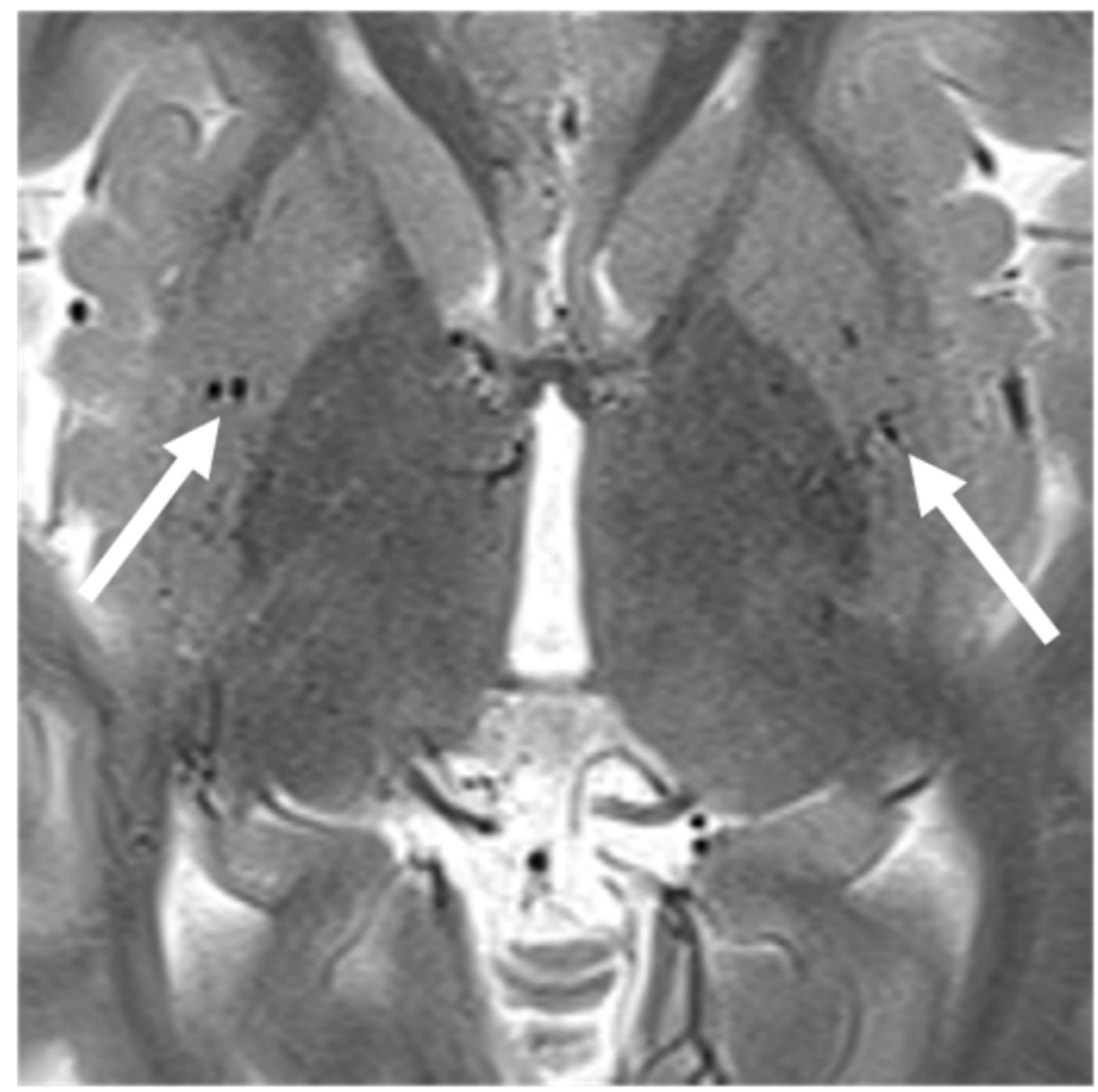

Fig. 2 


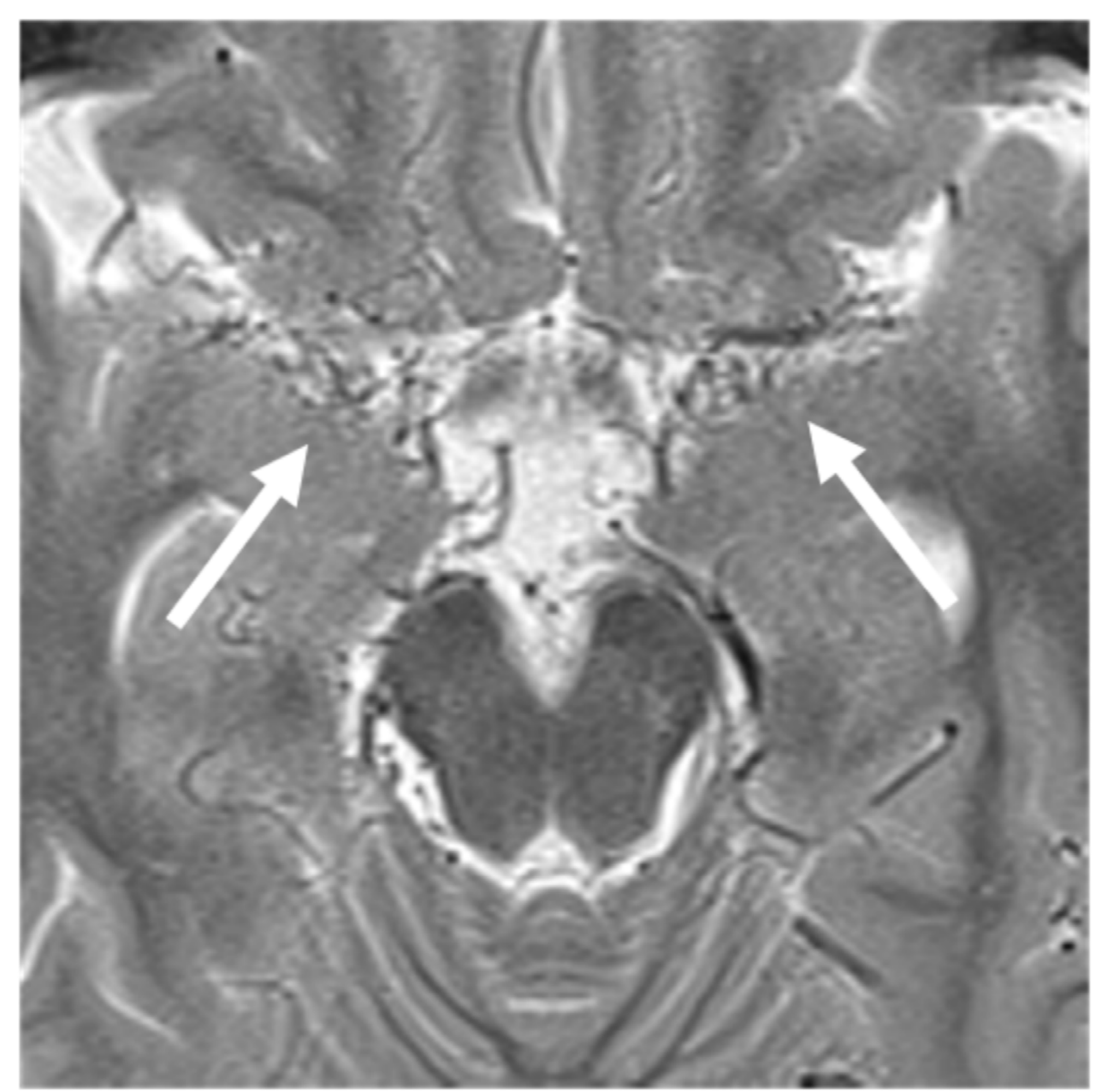

Fig. 3 


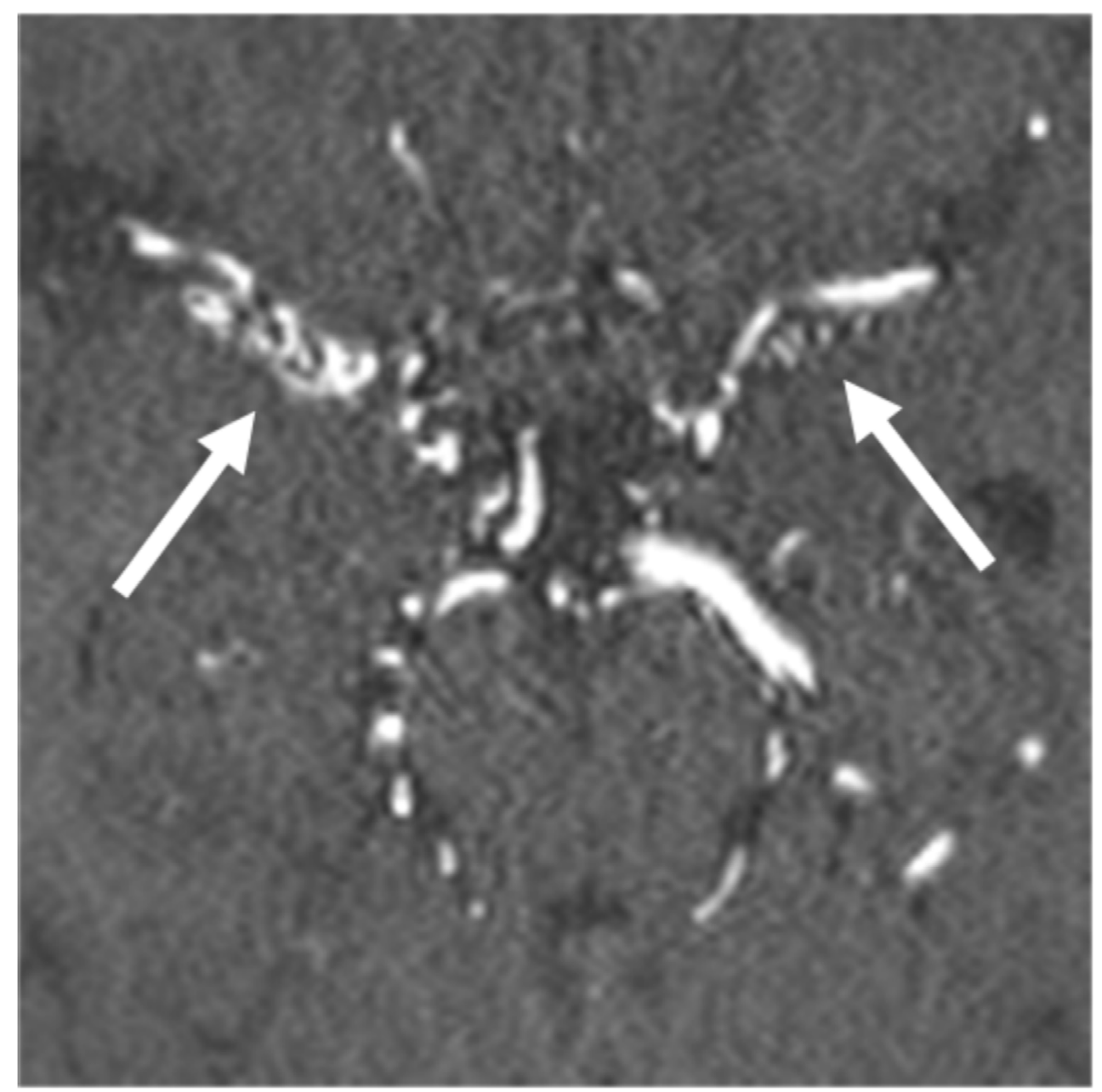

Fig. 4 


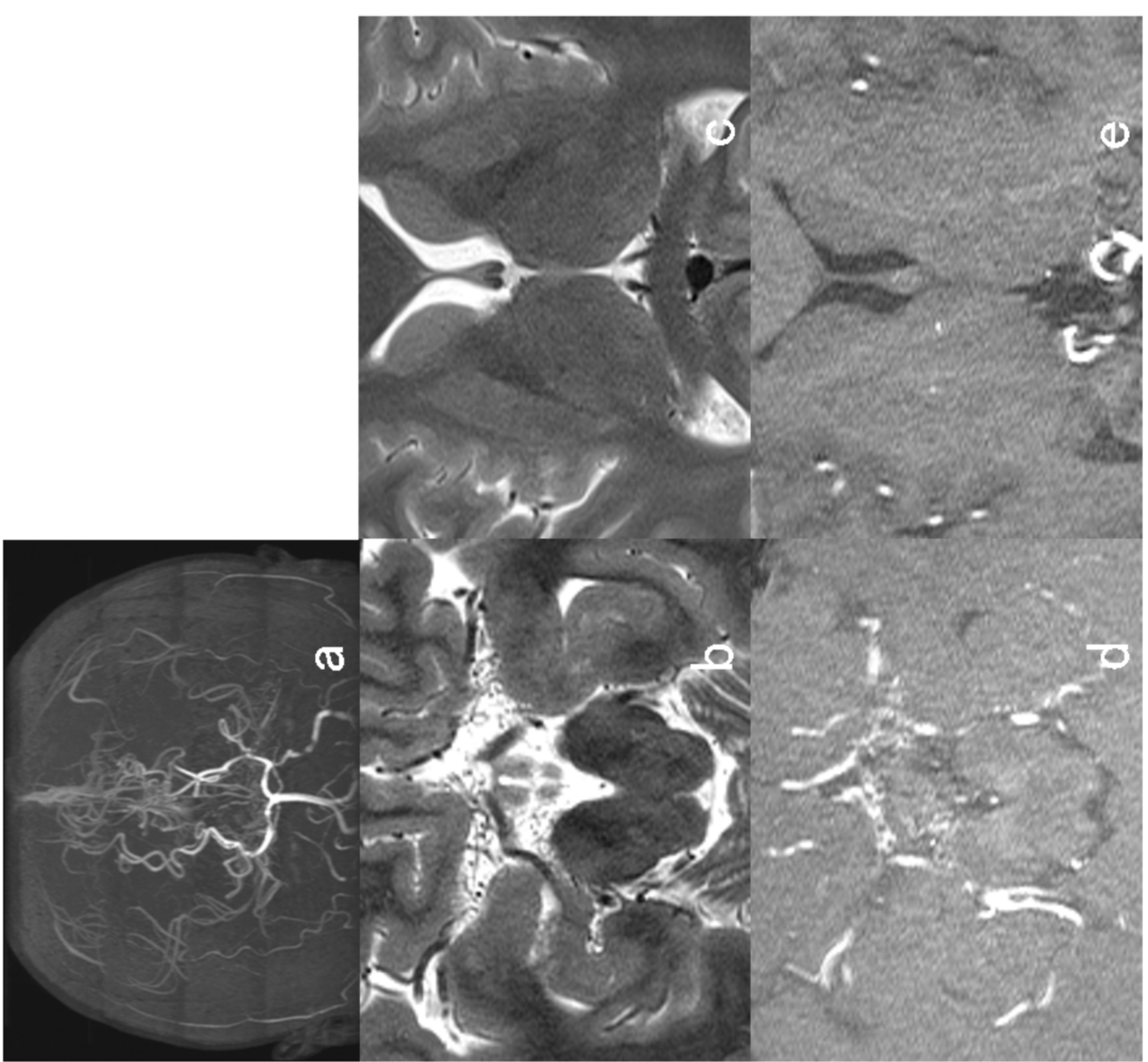

Fig. 5 


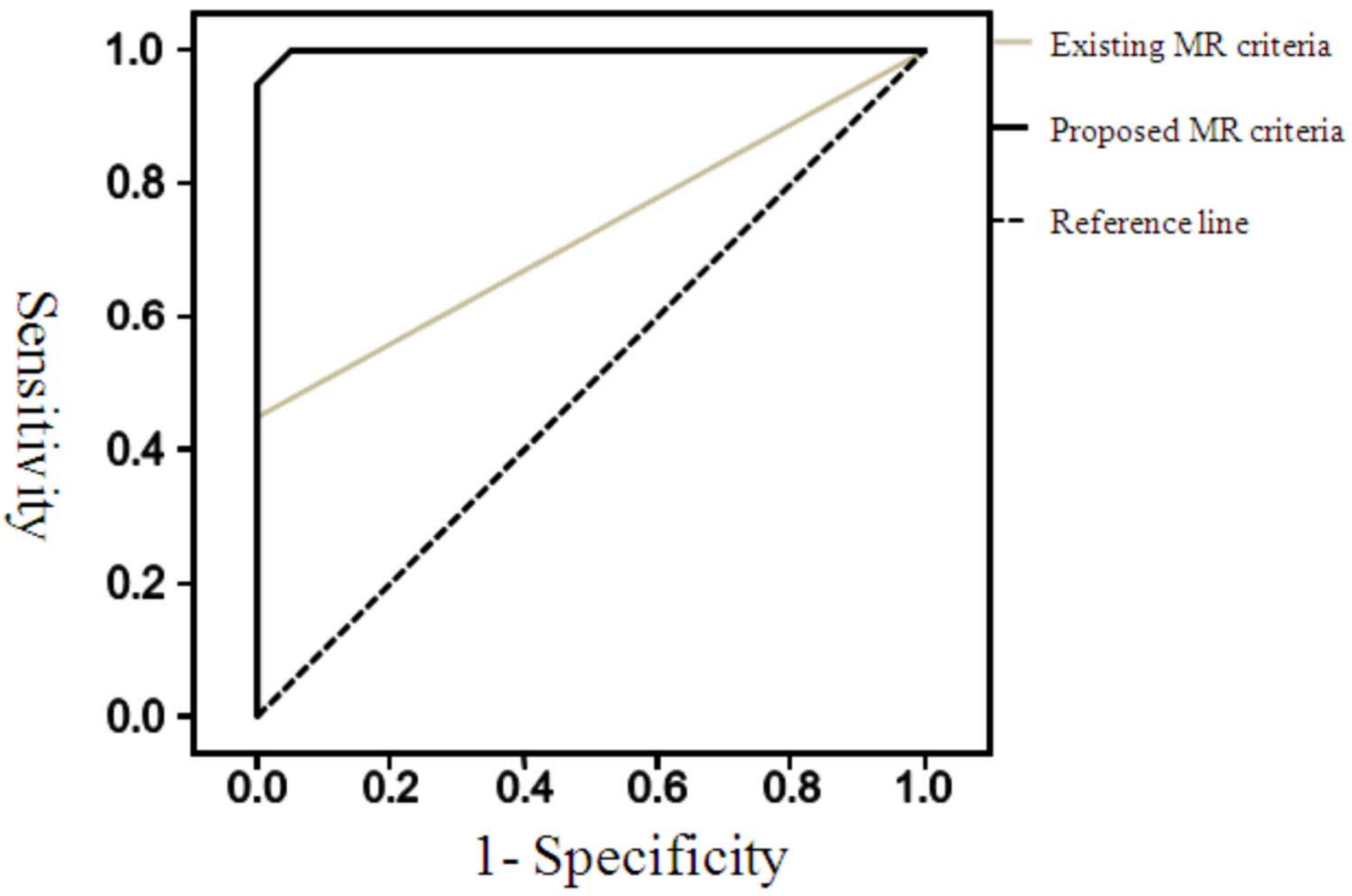

Fig. 6 